\title{
The Relative Toxicity of Road Salt Alternatives to Freshwater Mussels; Examining the Potential Risk of Eco-friendly De-icing Products to Sensitive Aquatic Species
}

Patricia Leigh Gillis ${ }^{1}$, Joseph Salerno ${ }^{1}$, C. James Bennett ${ }^{1}$, Yaryna Kudla ${ }^{1}$, Margot Smith ${ }^{2}$

\author{
${ }^{1}$ Aquatic Contaminants Research Division, Environment and Climate Change Canada, \\ Burlington, ON, Canada \\ ${ }^{2}$ Species at Risk Program, Department of Fisheries and Oceans, Burlington, ON, Canada
}

Corresponding Author

Patricia Gillis, patty.gillis@canada.ca, ORCID 0000-0003-3417-7966 


\section{Supporting Information for Publication}

Table S1. Sugar profile analysis (fructose, glucose, sucrose, maltose, lactose, total sugars; g/100 g) of (undiluted) de-icing products. Samples were analyzed by Silliker Canada Co. (Markham, ON) using HPLC (modified from the Association of Official Agricultural Chemists 982.14).

\begin{tabular}{cccc}
\hline Sugar Profile & $\begin{array}{c}\text { Brine } \\
(\mathbf{g} / \mathbf{1 0 0} \mathbf{g})\end{array}$ & $\begin{array}{c}\text { Brine-Beet Juice } \\
\mathbf{( g / 1 0 0 ~ g )}\end{array}$ & $\begin{array}{c}\text { Beet Juice } \\
\mathbf{( g / 1 0 0 ~ g )}\end{array}$ \\
\hline Fructose & $<0.2$ & $<0.2$ & $<0.2$ \\
Glucose & $<0.2$ & $<0.2$ & 0.29 \\
Sucrose & $<0.2$ & 1.1 & 6.59 \\
Maltose & $<0.5$ & $<0.5$ & $<0.5$ \\
Lactose & $<0.5$ & $<0.5$ & $<0.5$ \\
Total Sugars & $<1.6$ & 1.1 & 6.88 \\
\hline
\end{tabular}

Table S2. Physicochemical properties of water ( $n=4$, standard deviations (SD) in parentheses) from Environment and Climate Change Canada's (ECCC) Aquatic Life Research Facility used to hold gravid mussels. Analyses were conducted by ECCC's National Laboratory for Environmental Testing in Burlington, ON, Canada.

\begin{tabular}{cc}
\hline Physicochemical property & Mean value (SD) \\
\hline $\mathrm{Ca}^{2+}$ & $35.7(0.3) \mathrm{mg} / \mathrm{L}$ \\
$\mathrm{Mg}^{2+}$ & $9.0(0.07) \mathrm{mg} / \mathrm{L}$ \\
$\mathrm{K}^{+}$ & $1.7(0.02) \mathrm{mg} / \mathrm{L}$ \\
$\mathrm{Silica}^{+}$ & $1.3(0.04) \mathrm{mg} / \mathrm{L}$ \\
$\mathrm{Na}^{+}$ & $15.5(0.6) \mathrm{mg} / \mathrm{L}$ \\
$\mathrm{Cl}^{-}$ & $26.5(0.3) \mathrm{mg} / \mathrm{L}$ \\
$\mathrm{F}^{-}$ & $0.7(0.01) \mathrm{mg} / \mathrm{L}$ \\
$\mathrm{SO}_{4}{ }^{2-}$ & $26.9(0.1) \mathrm{mg} / \mathrm{L}$ \\
Dissolved inorganic carbon & $21.6(0.1) \mathrm{mg} / \mathrm{L}$ \\
Dissolved organic carbon & $0.9(0.0) \mathrm{mg} / \mathrm{L}$ \\
$\mathrm{NH}_{4}{ }^{+} / \mathrm{NH}_{3}$ & $0.017(0.001) \mathrm{mg} / \mathrm{L}$ \\
$\mathrm{NO}_{3}{ }^{-} \mathrm{NO}_{2}{ }^{-}$ & $0.78(0.23) \mathrm{mg} / \mathrm{L}$ \\
Soluble reactive phosphorus & $0.009(0.006) \mathrm{mg} / \mathrm{L}$ \\
Nitrogen, total $\mathrm{Kjeldahl}^{-}$ & $0.16(0.03) \mathrm{mg} / \mathrm{L}$ \\
Alkalinity $\left(\mathrm{CaCO}_{3}\right)$ & $90.35(0.64) \mathrm{mg} / \mathrm{L}$ \\
$\mathrm{pH}$ & $8.13(0.28)$ \\
Specific conductance $\left(25^{\circ} \mathrm{C}\right)$ & $316(4) \mu \mathrm{S} / \mathrm{cm}$ \\
$\mathrm{Hardness}$ & $126(0) \mathrm{mg} / \mathrm{L}$ \\
\hline
\end{tabular}


Table S3. Viability at 24 and $48 \mathrm{~h}$ ( $\mathrm{n}=1$ per treatment) and selected benchtop water chemistry of range finding and definitive exposures with glochidia and de-icing products. In Rangefinder \#1 (A) viability at test initiation $(\mathrm{t}=0 \mathrm{~h}$ ) was $98 \%$ (standard deviation $(\mathrm{SD})=0.82 \%, \mathrm{n}=3$ ). In Rangefinder \#2 (B), viability at $\mathrm{t}=0 \mathrm{~h}$ was $95.5 \%(\mathrm{SD}=1.1 \%, \mathrm{n}=3)$. In the Definitive test $(\mathrm{C})$, the viability at test initiation $(\mathrm{t}=0 \mathrm{~h})$ was $97 \%$ (standard deviation $=1.27 \%, \mathrm{n}=3$ ). Viability counts in rangefinder $\# 1$ and 2 have an $n=1$ and the definitive test has an $n=3$ (standard deviation). The $0 \%$ treatment is a reconstituted moderately hard water control. Results reported as a dash (-) were not determined*.

A

\begin{tabular}{|c|c|c|c|c|c|c|c|c|c|}
\hline \multirow[t]{2}{*}{ Treatment } & \multirow[t]{2}{*}{$\begin{array}{l}\text { Dilution } \\
(\%)\end{array}$} & \multicolumn{2}{|c|}{$\begin{array}{l}\text { Viability } \\
(\%)\end{array}$} & \multirow{2}{*}{$\begin{array}{c}\text { Cond } \\
(\mu \mathrm{S} / \mathrm{cm}) \\
48 \mathrm{~h}\end{array}$} & \multirow{2}{*}{$\begin{array}{c}\mathrm{Cl}^{-} \\
(\mathrm{mg} \mathrm{Cl} / \mathrm{L}) \\
48 \mathrm{~h}\end{array}$} & \multicolumn{2}{|c|}{$\begin{array}{c}\mathrm{DO} \\
(\mathrm{mg} / \mathrm{L})\end{array}$} & \multicolumn{2}{|c|}{$\begin{array}{c}\mathrm{NH}_{3} \\
(\mathrm{mg} \mathrm{N} / \mathrm{L})\end{array}$} \\
\hline & & $24 \mathrm{~h}$ & $48 \mathrm{~h}$ & & & $\mathbf{O} \mathbf{h}$ & $48 \mathrm{~h}$ & $\mathbf{0 ~ h}$ & $48 \mathrm{~h}$ \\
\hline \multirow{4}{*}{ Brine } & 0 & 77 & 81 & 443 & 69.8 & 8.56 & 8.73 & 0 & 0 \\
\hline & 0.01 & 94 & 93 & 316 & 28.6 & 8.53 & 8.77 & 0 & 0 \\
\hline & 0.1 & 88 & 89 & 728 & 237 & 8.64 & 8.87 & 0 & 0 \\
\hline & 1 & 7.9 & 9.1 & 4730 & 2540 & 8.61 & 8.70 & 0 & 0 \\
\hline \multirow{4}{*}{$\begin{array}{l}\text { Brine and Beet } \\
\text { Juice }\end{array}$} & 0 & 98 & 84 & 443 & 69.8 & 8.56 & 8.73 & 0 & 0 \\
\hline & 0.01 & 73 & 94 & 289 & 16.6 & 8.57 & 8.68 & 0 & 0 \\
\hline & 0.1 & 3.2 & 29 & 527 & 154 & 8.53 & 7.99 & 0 & 0 \\
\hline & 1 & 1.7 & 5.9 & 2807 & 1430 & 8.51 & 1.52 & 0 & 0 \\
\hline \multirow{4}{*}{ Beet Juice } & 0 & 85 & 96 & 443 & 69.8 & 8.56 & 8.73 & 0 & 0.25 \\
\hline & 0.01 & 93 & 92 & 301 & 17.5 & 8.55 & 8.20 & 0 & 0 \\
\hline & 0.1 & 2.3 & 7.3 & 652 & 102 & 8.41 & 0.54 & 0 & 0.25 \\
\hline & 1 & 3.3 & 1.4 & 3900 & 2280 & 5.81 & 0.26 & 0 & $-{ }^{*}$ \\
\hline
\end{tabular}

B

\begin{tabular}{|c|c|c|c|c|c|c|c|c|c|}
\hline \multirow[t]{2}{*}{ Treatment } & \multirow[t]{2}{*}{$\begin{array}{l}\text { Dilution } \\
(\%)\end{array}$} & \multicolumn{2}{|c|}{$\begin{array}{c}\text { Viability } \\
(\%)\end{array}$} & \multirow{2}{*}{$\begin{array}{c}\text { Cond } \\
(\mu \mathrm{S} / \mathrm{cm}) \\
48 \mathrm{~h} \\
\end{array}$} & \multirow{2}{*}{$\begin{array}{c}\mathrm{Cl}^{-} \\
(\mathrm{mg} \mathrm{Cl} / \mathrm{L}) \\
48 \mathrm{~h}\end{array}$} & \multicolumn{2}{|c|}{$\begin{array}{c}\mathrm{DO} \\
(\mathrm{mg} / \mathrm{L})\end{array}$} & \multicolumn{2}{|c|}{$\begin{array}{c}\mathrm{NH}_{3} \\
(\mathrm{mg} \mathrm{N} / \mathrm{L})\end{array}$} \\
\hline & & $24 \mathrm{~h}$ & $48 \mathrm{~h}$ & & & $\mathbf{0 ~ h}$ & $48 \mathrm{~h}$ & $\mathbf{0 ~ h}$ & $48 h$ \\
\hline \multirow{7}{*}{ Brine } & 0 & 98 & 96 & 297 & 19.9 & 8.75 & 8.82 & 0 & 0 \\
\hline & 0.01 & 93 & 92 & 336 & 47.4 & 8.92 & 9.04 & 0 & 0 \\
\hline & 0.05 & 97 & 92 & 510 & 128 & 8.96 & 9.07 & 0 & 0 \\
\hline & 0.1 & 92 & 90 & 726 & 227 & 8.95 & 9.08 & 0 & 0 \\
\hline & 0.5 & 30 & 70 & 2492 & 1050 & 9.06 & 9.05 & 0 & 0 \\
\hline & 1 & 6.3 & 17 & 4600 & 2390 & 8.95 & 9.01 & 0 & 0 \\
\hline & 2 & 7.9 & 3.3 & 8750 & 4540 & 9.03 & 8.96 & 0 & 0 \\
\hline \multirow{6}{*}{$\begin{array}{l}\text { Brine and Beet } \\
\text { Juice }\end{array}$} & 0.01 & 91 & 94 & 330 & 28.4 & 8.96 & 9 & 0 & 0 \\
\hline & 0.05 & 96 & 86 & 516 & 101 & 8.93 & 8.38 & 0 & 0.25 \\
\hline & 0.1 & 35 & 21 & 735 & 169 & 8.87 & 7.7 & 0 & 0.5 \\
\hline & 0.5 & 32 & 1.2 & 2466 & 669 & 8.79 & 1.21 & 0 & 0.5 \\
\hline & 1 & 16 & 1.5 & 4540 & 1390 & 8.53 & 0.53 & 0.25 & 0.5 \\
\hline & 2 & 0.5 & 20 & 8590 & 2680 & 8.10 & 0.68 & 0.25 & 0.25 \\
\hline \multirow{6}{*}{ Beet Juice } & 0.01 & 95 & 89 & 336 & 24.7 & 8.9 & 8.21 & 0 & 0.25 \\
\hline & 0.05 & 94 & 81 & 460 & 63.3 & 9.06 & 3.59 & 0 & 0.5 \\
\hline & 0.1 & 5.4 & 1.6 & 619 & 72.8 & 8.83 & 0.82 & 0 & 0.5 \\
\hline & 0.5 & 1.6 & 5.8 & 1975 & 435 & 7.44 & 0.68 & - & - \\
\hline & 1 & 0.9 & 9.2 & 3610 & 1450 & 4.48 & 0.27 & - & - \\
\hline & 2 & 1.0 & 9.4 & 6650 & 3420 & 0.74 & 0.13 & - & - \\
\hline
\end{tabular}

*Ammonia could not be measured due to colour interference from the beet juice containing de-icing product (see Figure S1 for colour) 
Table S3 Continued.

C

\begin{tabular}{|c|c|c|c|c|c|c|c|c|c|}
\hline \multirow[t]{2}{*}{ Treatment } & \multirow[t]{2}{*}{$\begin{array}{c}\text { Dilution } \\
(\%)\end{array}$} & \multicolumn{2}{|c|}{$\begin{array}{c}\text { Viability } \\
\text { (\%) }\end{array}$} & \multirow{2}{*}{$\begin{array}{c}\text { Cond } \\
(\mu \mathrm{S} / \mathrm{cm}) \\
48 \mathrm{~h}\end{array}$} & \multirow{2}{*}{$\begin{array}{c}\mathrm{Cl}^{-} \\
(\mathrm{mg} \mathrm{Cl} / \mathrm{L}) \\
48 \mathrm{~h}\end{array}$} & \multicolumn{2}{|c|}{$\begin{array}{c}\text { DO } \\
(\mathrm{mg} / \mathrm{L})\end{array}$} & \multicolumn{2}{|c|}{$\begin{array}{c}\mathrm{NH}_{3} \\
(\mathrm{mg} \mathrm{N} / \mathrm{L})\end{array}$} \\
\hline & & $24 \mathrm{~h}$ & $48 \mathrm{~h}$ & & & $\mathbf{O} \mathbf{h}$ & $48 \mathrm{~h}$ & $\mathbf{O} \mathbf{h}$ & $48 \mathrm{~h}$ \\
\hline \multirow[t]{8}{*}{ Brine } & 0 & $92(1.0)$ & $94(2.1)$ & 267.5 & 3.48 & 8.68 & 8.76 & 0 & 0 \\
\hline & 0.01 & $96(3.3)$ & $96(1.4)$ & 326 & 13.7 & 8.97 & 9.13 & 0 & 0 \\
\hline & 0.1 & $90(1.8)$ & $86(8.5)$ & 777 & 229 & 8.91 & 9.09 & 0 & 0 \\
\hline & 0.3 & $64(2.6)$ & $66(15)$ & 1678 & 693 & 8.85 & 9.02 & 0 & 0 \\
\hline & 0.5 & $52(11)$ & $45(4.4)$ & 2511 & 990 & 9.02 & 9.08 & 0 & 0 \\
\hline & 0.75 & $42(2.7)$ & $25(25)$ & 3660 & 1730 & 8.91 & 9.22 & 0 & 0 \\
\hline & 1 & $2.0(1.3)$ & $23(11)$ & 4620 & 2150 & 8.90 & 9.20 & 0 & 0 \\
\hline & 2 & $0.5(1.0)$ & $0.0(0.0)$ & 8720 & 4980 & 9.02 & 9.15 & 0 & 0 \\
\hline \multirow[t]{8}{*}{ Brine-Beet Juice } & 0 & $96(1.4)$ & $93(3.9)$ & 270.4 & 3.81 & 8.82 & 8.81 & 0 & 0 \\
\hline & 0.01 & $95(3.3)$ & $93(3.4)$ & 311 & 9.31 & 8.92 & 8.96 & 0 & 0 \\
\hline & 0.03 & $79(13)$ & 59 (19) & 401 & 66.1 & 8.97 & 8.77 & 0 & 0 \\
\hline & 0.05 & 49 (19) & $25(25)$ & 546 & 109 & 8.99 & 7.82 & 0 & 0 \\
\hline & 0.1 & $11(7.5)$ & $12(10)$ & 727 & 175 & 8.8 & 6.61 & 0 & 0 \\
\hline & 0.5 & $2.2(3.9)$ & $4.6(5.4)$ & 2498 & 1090 & 8.83 & 0.76 & 0.25 & 0.25 \\
\hline & 1 & $3.9(5.2)$ & $0.0(0.0)$ & 4530 & 1710 & 8.68 & 0.48 & 0.25 & 0.5 \\
\hline & 2 & $4.2(4.8)$ & $0.4(0.4)$ & 8380 & 3370 & 8.17 & 0.33 & 0.5 & - \\
\hline \multirow[t]{8}{*}{ Beet Juice } & 0 & $96(2.7)$ & $95(2.5)$ & 270.4 & 3.81 & 8.82 & 8.81 & 0 & 0 \\
\hline & 0.01 & $93(5.6)$ & $93(6.0)$ & 302 & 5.81 & 9.02 & 8.21 & 0 & 0 \\
\hline & 0.03 & $16(15)$ & $17(4.6)$ & 382 & 21.6 & 8.86 & 5.81 & 0 & 0.25 \\
\hline & 0.05 & $6.5(9.9)$ & $9.5(5.7)$ & 447 & 24.3 & 8.81 & 3.73 & 0 & 0.5 \\
\hline & 0.1 & $4.5(8.6)$ & $5.0(3.7)$ & 584 & 37 & 8.64 & 0.43 & 0 & 0.5 \\
\hline & 0.5 & $0.2(0.3)$ & $4.3(3.9)$ & 1861 & 695 & 7.15 & 0.37 & 0.5 & $-*$ \\
\hline & 1 & $0.3(0.7)$ & $0.8(0.9)$ & 3290 & 1320 & 4.83 & 0.31 & - & - \\
\hline & 2 & $2.0(4.0)$ & $1.0(1.9)$ & 6010 & 2820 & 1.11 & 0.31 & - & - \\
\hline
\end{tabular}

*Ammonia could not be measured due to colour interference from the beet juice containing de-icing product (see Figure S1) 
Table S4. Benchtop water chemistry summary for the definitive exposure with freshwater mussel glochidia and de-icing products. Results are reported for $\mathrm{n}=3$, with standard deviation, where a dash (-) indicates data not available*. The $0 \%$ treatment is a reconstituted moderatelyhard water control.

\begin{tabular}{|c|c|c|c|c|c|c|}
\hline Treatment & Dilution (\%) & pH & $\begin{array}{c}\text { Cond } \\
(\mu \mathrm{S} / \mathrm{cm})\end{array}$ & $\begin{array}{c}\mathrm{Cl}^{-} \\
(\mathrm{mg} \mathrm{Cl} / \mathrm{L})\end{array}$ & $\begin{array}{c}\text { DO } \\
(\mathrm{mg} / \mathrm{L})\end{array}$ & $\begin{array}{c}\mathrm{NH}_{3} \\
(\mathrm{mg} \mathrm{N} / \mathrm{L})\end{array}$ \\
\hline \multirow{8}{*}{ Brine } & 0.0 & $8.24(0.00)$ & $255(10)$ & $2.6(0.9)$ & $8.71(0.03)$ & $0.0(0.0)$ \\
\hline & 0.01 & $8.25(0.00)$ & $306(14)$ & $11.4(2.9)$ & $9.07(0.07)$ & $0.0(0.0)$ \\
\hline & 0.1 & $8.24(0.00)$ & $732(33)$ & $225(54)$ & $9.05(0.11)$ & $0.0(0.0)$ \\
\hline & 0.3 & $8.20(0.01)$ & $1614(47)$ & $594(106)$ & $8.98(0.09)$ & $0.0(0.0)$ \\
\hline & 0.5 & $8.19(0.01)$ & $2464(35)$ & $876(111)$ & $9.07(0.03)$ & $0.0(0.0)$ \\
\hline & 0.75 & $8.17(0.00)$ & $3560(82)$ & $1733(61)$ & $9.12(0.15)$ & $0.0(0.0)$ \\
\hline & 1.0 & $8.13(0.04)$ & $4560(49)$ & $2190(158)$ & $9.12(0.16)$ & $0.0(0.0)$ \\
\hline & 2.0 & $8.14(0.02)$ & $8560(131)$ & $4160(700)$ & $9.11(0.06)$ & $0.0(0.0)$ \\
\hline \multirow{8}{*}{$\begin{array}{l}\text { Brine-Beet } \\
\text { Juice }\end{array}$} & 0.0 & $8.25(0.01)$ & $258(9)$ & $2.4(1.1)$ & $8.79(0.03)$ & $0.0(0.0)$ \\
\hline & 0.01 & $8.27(0.02)$ & $295(12)$ & $7.1(2.0)$ & $9.03(0.13)$ & $0.0(0.0)$ \\
\hline & 0.03 & $8.25(0.04)$ & $268(166)$ & $40.3(20)$ & $8.94(0.12)$ & $0.0(0.0)$ \\
\hline & 0.05 & $8.19(0.11)$ & $513(31)$ & $122(12)$ & $8.64(0.58)$ & $0.0(0.0)$ \\
\hline & 0.1 & $8.11(0.18)$ & $708(16)$ & $183(6)$ & $8.17(1.11)$ & $0.0(0.0)$ \\
\hline & 0.5 & $8.00(0.29)$ & $2431(49)$ & $1060(218)$ & $6.24(3.88)$ & $0.3(0.0)$ \\
\hline & 1.0 & $7.92(0.38)$ & $4447(62)$ & $2053(640)$ & $6.02(3.92)$ & $0.4(0.1)$ \\
\hline & 2.0 & $7.82(0.44)$ & $8280(78)$ & $4017(1254)$ & $5.69(3.80)$ & $0.5(0.0)$ \\
\hline \multirow{8}{*}{ Beet Juice } & 0.0 & $8.25(0.01)$ & $258(9)$ & $2.4(1.1)$ & $8.79(0.03)$ & $0.0(0.0)$ \\
\hline & 0.01 & $8.18(0.07)$ & $293(7)$ & $6.5(2.6)$ & $8.80(0.42)$ & $0.0(0.0)$ \\
\hline & 0.03 & $8.10(0.23)$ & $359(17)$ & $24(14)$ & $7.90(1.48)$ & $0.1(0.1)$ \\
\hline & 0.05 & $8.07(0.31)$ & $425(17)$ & $40(26)$ & $7.19(2.45)$ & $0.2(0.2)$ \\
\hline & 0.1 & $8.06(0.41)$ & $570(10)$ & $53.1(11.8)$ & $5.98(3.93)$ & $0.3(0.2)$ \\
\hline & 0.5 & $8.19(0.37)$ & $1810(39)$ & $690(222)$ & $5.30(3.52)$ & $0.5(0.0)$ \\
\hline & 1.0 & $8.25(0.35)$ & $3250(29)$ & $1453(411)$ & $3.92(2.66)$ & - \\
\hline & 2.0 & $8.13(0.37)$ & $5950(1240)$ & $2937(1087)$ & $1.44(1.08)$ & - \\
\hline
\end{tabular}

*Ammonia could not be measured due to colour interference from the beet juice containing de-icing product (see Figure S1). 
Table S5. Major ions (mg/L), biological oxygen demand (BOD), carbon (mg/L), and ammonia $(\mathrm{mg} \mathrm{N} / \mathrm{L})$ at test initiation $(0 \mathrm{~h})$ and completion $(48 \mathrm{~h})$ of the definitive exposure with freshwater mussel glochidia and de-icing products definitive tests. Results reported by ECCC's National Laboratory for Environmental Testing (NLET) (Burlington, ON, Canada). Results reported as a dash (-) were not submitted for analysis. The $0 \%$ treatment is a reconstituted moderately-hard water control.

\begin{tabular}{|c|c|c|c|c|c|c|c|c|c|c|c|c|c|c|}
\hline Treatment & $\begin{array}{c}\text { Time } \\
\text { (h) }\end{array}$ & $\begin{array}{c}\text { Dilution } \\
(\%)\end{array}$ & $\begin{array}{c}\mathrm{F}^{-} \\
(\mathrm{mg} / \mathrm{L})\end{array}$ & $\underset{(\mathrm{mg} / \mathrm{L})}{\mathrm{Cl}^{-}}$ & $\begin{array}{c}\mathrm{SO}_{4}{ }^{2-} \\
(\mathrm{mg} / \mathrm{L})\end{array}$ & $\begin{array}{c}\text { NH } \\
(\mathrm{mg} \mathrm{N} / \mathrm{L})\end{array}$ & $\begin{array}{c}\mathrm{Ca}^{2+} \\
(\mathrm{mg} / \mathrm{L})\end{array}$ & $\begin{array}{c}\mathrm{Mg}^{2+} \\
(\mathrm{mg} / \mathrm{L})\end{array}$ & $\begin{array}{c}\mathrm{Na}^{+} \\
(\mathrm{mg} / \mathrm{L})\end{array}$ & $\begin{array}{c}\mathbf{K}^{+} \\
(\mathbf{m g} / \mathbf{L})\end{array}$ & $\begin{array}{c}\mathrm{Si} \\
(\mathrm{mg} / \mathrm{L})\end{array}$ & BOD & $\begin{array}{c}\mathrm{DOC} \\
(\mathrm{mg} / \mathrm{L})\end{array}$ & $\begin{array}{c}\text { DIC } \\
(\mathrm{mg} / \mathrm{L})\end{array}$ \\
\hline \multirow{16}{*}{ Brine } & \multirow{8}{*}{$\mathrm{T}=0$} & 0 & $<0.01$ & 5.73 & 80.3 & 0.009 & 14.2 & 11.7 & 26.5 & 2.65 & 0.02 & $<2.0$ & - & - \\
\hline & & 0.01 & $<0.05$ & 22.9 & 80.9 & 0.01 & 14.3 & 11.7 & 37.8 & 2.67 & 0.02 & $<2.0$ & - & - \\
\hline & & 0.1 & $<0.05$ & 174 & 83.6 & - & 14.8 & 11.7 & 137 & 2.8 & $<0.01$ & - & - & - \\
\hline & & 0.3 & $<0.05$ & 504 & 87.8 & - & 17.0 & 11.9 & 368 & 2.96 & $<0.01$ & - & - & - \\
\hline & & 0.5 & $<0.10$ & 842 & 92.5 & 0.016 & 18.8 & 11.9 & 594 & 3.00 & $<0.01$ & 2.1 & - & - \\
\hline & & 0.75 & $<0.20$ & 1250 & 96.8 & - & 21.0 & 11.8 & 878 & 3.32 & $<0.01$ & - & - & - \\
\hline & & 1 & $<0.50$ & 1690 & 101 & - & 23.5 & 12.0 & 1160 & 3.28 & $<0.01$ & - & - & - \\
\hline & & 2 & $<1.00$ & 3420 & 123 & 0.022 & 32.8 & 12.1 & 2270 & 3.87 & 0.02 & 2.1 & - & - \\
\hline & \multirow{8}{*}{$\mathrm{T}=48$} & 0 & $<0.01$ & 12.9 & 81.6 & 0.027 & 14.5 & 12.2 & 27.5 & $-*$ & 0.35 & 4.5 & 0.7 & 0.7 \\
\hline & & 0.01 & $<0.05$ & 24.1 & 83.6 & 0.028 & 14.6 & 12.4 & 39.5 & - & 0.36 & 4.0 & - & - \\
\hline & & 0.1 & $<0.05$ & 201 & 85.5 & - & 15.3 & 12.5 & 144 & - & 0.21 & - & - & - \\
\hline & & 0.3 & $<0.05$ & 543 & 89.9 & - & 17.3 & 12.4 & 376 & - & 0.38 & - & - & - \\
\hline & & 0.5 & $<0.10$ & 865 & 94.5 & 0.035 & 19.6 & 12.6 & 625 & - & 0.37 & 3.3 & - & - \\
\hline & & 0.75 & $<0.20$ & 1320 & 101 & - & 22.2 & 12.7 & 923 & - & 0.14 & - & - & - \\
\hline & & 1 & $<0.50$ & 1740 & 103 & - & 24.5 & 12.6 & 1210 & - & 0.41 & - & - & - \\
\hline & & 2 & $<1.00$ & 3520 & 125 & 0.046 & 34.8 & 12.9 & 2410 & - & 0.42 & 4.6 & - & - \\
\hline \multirow{16}{*}{ Brine-Beet Juice } & \multirow{8}{*}{$\mathrm{T}=0$} & 0 & $<0.01$ & 5.11 & 81.6 & 0.019 & 14.4 & 12.2 & 27.3 & 14.7 & 0.26 & 4.8 & - & - \\
\hline & & 0.01 & $<0.05$ & 16.3 & 82.6 & 0.021 & 14.1 & 12.1 & 34.5 & 4.58 & 0.03 & 9.0 & - & - \\
\hline & & 0.03 & $<0.05$ & 39.3 & 84.2 & - & 14.2 & 12.0 & 49.6 & 7.14 & 0.02 & - & - & - \\
\hline & & 0.05 & $<0.05$ & 77.7 & 85.3 & - & 14.4 & 12.0 & 75.9 & 12.1 & 0.04 & - & - & - \\
\hline & & 0.1 & $<0.10$ & 150 & 87.5 & 0.038 & 15.3 & 12.5 & 126 & 22.0 & 0.05 & 49.2 & - & - \\
\hline & & 0.5 & $<0.20$ & 730 & 105 & - & 19.3 & 12.9 & 677 & 101 & 0.18 & - & - & - \\
\hline & & 1 & $<0.50$ & 1480 & 130 & - & 23.4 & 12.9 & 1020 & 215 & 0.33 & - & - & - \\
\hline & & 2 & $<1.00$ & 3070 & 181 & 0.286 & 32.4 & 13.6 & 2070 & 397 & 0.61 & 1210 & - & - \\
\hline & \multirow{8}{*}{$\mathrm{T}=48$} & 0 & $<0.01$ & 16.3 & 83.9 & 0.028 & 14.4 & 12.2 & 27.3 & 14.7 & 0.26 & 8.4 & 0.4 & 12.8 \\
\hline & & 0.01 & $<0.05$ & 16.9 & 85.4 & 0.034 & 14.6 & 12.5 & 35.9 & 41.0 & 0.27 & 12 & 3.4 & 12.8 \\
\hline & & 0.03 & $<0.05$ & 62.4 & 86.2 & - & 14.8 & 12.3 & 52.0 & 32.3 & 0.27 & - & - & - \\
\hline & & 0.05 & $<0.05$ & 104 & 88.4 & - & 15.0 & 12.3 & 79.5 & 39.4 & 0.13 & - & - & - \\
\hline & & 0.1 & $<0.10$ & 156 & 90.9 & 0.17 & 15.6 & 12.8 & 135 & 24.8 & 0.3 & 75 & 38.1 & 15.5 \\
\hline & & 0.5 & $<0.20$ & 783 & 110 & - & 19.0 & 13.2 & 555 & 138 & 0.24 & - & - & - \\
\hline & & 1 & $<0.50$ & 1530 & 135 & - & 24.0 & 13.2 & 1090 & 227 & 0.26 & - & - & - \\
\hline & & 2 & $<1.00$ & 3130 & 187 & 0.47 & 33.2 & 13.7 & 2170 & 421 & 0.5 & 1180 & 885 & 30.2 \\
\hline \multirow{16}{*}{ Beet Juice } & \multirow{8}{*}{$\mathrm{T}=0$} & 0 & $<0.01$ & 5.11 & 81.6 & 0.019 & 14.4 & 12.2 & 27.3 & 14.7 & 0.26 & 4.8 & - & - \\
\hline & & 0.01 & $<0.05$ & 8.57 & 84.0 & 0.027 & 14.2 & 12.3 & 32.4 & 9.95 & 0.03 & 23.1 & 15.9 & 13.2 \\
\hline & & 0.03 & $<0.05$ & 15.6 & 85.7 & - & 14.3 & 12.3 & 42.0 & 24.9 & 0.04 & - & - & - \\
\hline & & 0.05 & $<0.05$ & 22.6 & 87.1 & - & 14.3 & 12.2 & 51.3 & 39.8 & 0.05 & - & - & - \\
\hline & & 0.1 & $<0.10$ & 40.7 & 93.0 & 0.085 & 14.6 & 12.1 & 75.1 & 78.1 & 0.09 & 192 & 182 & 30.9 \\
\hline & & 0.5 & $<0.20$ & 185 & 132 & - & 18.3 & 13.2 & 271 & 371 & 0.33 & - & - & - \\
\hline & & 1 & $<0.50$ & 375 & 185 & - & 21.2 & 13.4 & 610 & 740 & 0.62 & - & - & - \\
\hline & & 2 & $<1.00$ & 742 & 281 & 0.456 & 27.6 & 14.7 & 967 & 1530 & 1.15 & 4740 & 4260 & 30.2 \\
\hline & \multirow{8}{*}{$\mathrm{T}=48$} & 0 & $<0.01$ & 16.3 & 83.9 & 0.028 & 14.4 & 12.2 & 27.3 & 14.7 & 0.26 & 8.4 & 0.4 & 12.8 \\
\hline & & 0.01 & $<0.05$ & 8.88 & 86.6 & 0.039 & 14.3 & 12.3 & 32.1 & 9.94 & 0.21 & 32.7 & 15.9 & 13.2 \\
\hline & & 0.03 & $<0.05$ & 33.8 & 88.5 & - & 14.9 & 12.4 & 42.2 & 42.8 & 0.44 & - & - & - \\
\hline & & 0.05 & $<0.05$ & 35.1 & 90.9 & - & 14.9 & 12.3 & 51.7 & 53.3 & 0.33 & - & - & - \\
\hline & & 0.1 & $<0.10$ & 51.9 & 94.7 & 0.596 & 15.0 & 12.5 & 75.8 & 91.4 & 0.16 & 246 & 182 & 30.9 \\
\hline & & 0.5 & $<0.20$ & 206 & 141 & - & 18.4 & 13.3 & 278 & 414 & 0.2 & - & - & - \\
\hline & & 1 & $<0.50$ & 396 & 193 & - & 21.5 & 13.5 & 525 & 799 & 0.53 & - & - & - \\
\hline & & 2 & $<1.00$ & 808 & 313 & 0.699 & 30.2 & 15.7 & 1110 & 1720 & 1.48 & 4410 & 4260 & 30.2 \\
\hline
\end{tabular}

*Statistical analysis involving potassium for the brine exposure only used concentrations at $\mathrm{t}=0$.

Since the $\mathrm{t}=48 \mathrm{~h}$ potassium data did not reflect the same trend based on previous analytical water quality testing of the brine product, this sampling event was omitted. Interference between samples of high to those of low potassium concentration may have been a factor during analysis. 
Table S6. Dissolved metals $(\mu \mathrm{g} / \mathrm{L})$ at test completion $(48 \mathrm{~h})$ in selected treatments of the definitive exposure with freshwater mussel glochidia and de-icing products. Results reported by ECCC's National Laboratory for Environmental Testing (NLET) (Burlington, ON, Canada). The 0\% treatment is a reconstituted moderately hard water control.

\begin{tabular}{|c|c|c|c|c|c|c|c|c|c|c|c|c|}
\hline \multirow{2}{*}{ Dissolved Metal $(\mu \mathrm{g} / \mathrm{L})$} & \multicolumn{4}{|c|}{ Brine } & \multicolumn{4}{|c|}{ Brine-Beet Juice } & \multicolumn{4}{|c|}{ Beet Juice } \\
\hline & $0 \%$ & $0.01 \%$ & $0.5 \%$ & $2 \%$ & $0 \%$ & $0.01 \%$ & $0.1 \%$ & $2 \%$ & $0 \%$ & $0.01 \%$ & $0.1 \%$ & $2 \%$ \\
\hline Aluminum & 1.5 & 1.2 & 1.7 & 1.6 & 5.8 & 8.7 & 5.4 & 32.5 & 5.8 & 7.9 & $<5.0$ & 104 \\
\hline Antimony & 0.010 & 0.005 & 0.0019 & 0.057 & 0.007 & 0.011 & 0.050 & 0.760 & 0.007 & 0.025 & 0.115 & 2.31 \\
\hline Arsenic & 0.01 & 0.01 & 0.02 & 0.03 & $<0.01$ & $<0.01$ & 0.02 & 0.11 & $<0.01$ & 0.01 & $<0.10$ & 0.53 \\
\hline Barium & 0.19 & 0.18 & 0.43 & 1.19 & 0.22 & 0.23 & 0.39 & 1.64 & 0.22 & 0.20 & $<0.50$ & 1.84 \\
\hline Beryllium & $<0.001$ & $<0.001$ & $<0.001$ & $<0.001$ & $<0.001$ & 0.002 & $<0.001$ & $<0.010$ & $<0.001$ & $<0.001$ & $<0.010$ & $<0.010$ \\
\hline Bismuth & $<0.001$ & $<0.001$ & $<0.001$ & 0.002 & $<0.001$ & $<0.001$ & $<0.001$ & $<0.010$ & $<0.001$ & $<0.001$ & $<0.010$ & $<0.010$ \\
\hline Boron & 13.8 & 13.3 & 18.3 & 28.9 & 11.4 & 11.0 & 15.3 & 39.3 & 11.4 & 10.2 & 14.5 & 99.5 \\
\hline Cadmium & 0.003 & 0.001 & 0.003 & 0.009 & 0.005 & 0.002 & 0.002 & 0.010 & 0.005 & 0.002 & $<0.010$ & $<0.010$ \\
\hline Cerium & 0.002 & 0.002 & 0.003 & 0.004 & 0.003 & 0.004 & 0.003 & 0.013 & 0.003 & 0.003 & $<0.010$ & 0.028 \\
\hline Cesium & 0.002 & 0.002 & 0.002 & 0.006 & 0.002 & 0.002 & 0.019 & 0.345 & 0.002 & 0.008 & 0.071 & 1.29 \\
\hline Chromium & 0.02 & 0.02 & 0.03 & 0.06 & 0.05 & 0.08 & 0.12 & 1.12 & 0.05 & 0.09 & $<0.20$ & 4.54 \\
\hline Cobalt & 0.003 & 0.005 & 0.008 & 0.030 & 0.006 & 0.028 & 0.254 & 4.85 & 0.006 & 0.113 & 1.06 & 22.6 \\
\hline Copper & 0.54 & 0.48 & 0.48 & 0.62 & 0.40 & 0.49 & 0.86 & 2.71 & 0.40 & 0.57 & 0.71 & 5.06 \\
\hline Gallium & 0.001 & 0.001 & 0.001 & 0.001 & 0.002 & 0.004 & 0.007 & 0.149 & 0.002 & 0.006 & 0.030 & 0.525 \\
\hline Iron & 1.6 & 1.6 & 21.4 & 95.6 & 0.7 & 1.7 & 15.6 & 319 & 0.7 & 6.1 & 58.4 & 1320 \\
\hline Lanthanum & $<0.001$ & 0.001 & 0.002 & 0.002 & 0.002 & 0.003 & 0.002 & $<0.010$ & 0.002 & 0.002 & $<0.010$ & 0.011 \\
\hline Lead & 0.136 & 0.055 & 0.139 & 0.438 & 0.033 & 0.043 & 0.122 & 0.919 & 0.033 & 0.072 & 0.183 & 0.745 \\
\hline Lithium & 0.02 & 0.03 & 0.36 & 1.11 & 0.05 & 0.05 & 0.20 & 3.28 & 0.05 & 0.09 & 0.45 & 9.38 \\
\hline Manganese & 0.18 & 0.18 & 0.85 & 3.07 & 0.09 & 0.16 & 1.38 & 24.5 & 0.09 & 0.39 & 4.12 & 78.1 \\
\hline Molybdenum & 0.016 & 0.014 & 0.026 & 0.053 & 0.019 & 0.034 & 0.268 & 2.13 & 0.019 & 0.129 & 0.848 & 5.09 \\
\hline Nickel & 0.23 & 0.22 & 0.24 & 0.36 & 0.24 & 0.35 & 1.49 & 24.0 & 0.24 & 0.75 & 5.84 & 107 \\
\hline Niobium & $<0.002$ & $<0.002$ & $<0.002$ & 0.003 & $<0.002$ & $<0.002$ & 0.004 & $<0.020$ & $<0.002$ & $<0.002$ & $<0.020$ & 0.053 \\
\hline Platinum & $<0.001$ & $<0.001$ & $<0.001$ & $<0.001$ & $<0.001$ & $<0.001$ & $<0.001$ & $<0.010$ & $<0.001$ & $<0.001$ & $<0.010$ & 0.019 \\
\hline Rubidium & 0.244 & 0.324 & 0.344 & 0.607 & 0.344 & 1.05 & 12.8 & 249 & 0.344 & 5.28 & 50.9 & 1080 \\
\hline Selenium & $<0.01$ & $<0.01$ & 0.01 & 0.05 & 0.01 & 0.01 & 0.09 & 1.50 & 0.01 & 0.04 & 0.29 & 6.40 \\
\hline Silver & 0.021 & 0.010 & 0.015 & 0.046 & 0.025 & $<0.001$ & $<0.001$ & $<0.010$ & 0.025 & $<0.001$ & $<0.010$ & 0.183 \\
\hline Strontium & 2.79 & 3.78 & 63.0 & 269 & 2.98 & 3.52 & 12.7 & 191 & 2.98 & 3.30 & 7.37 & 97.1 \\
\hline Thallium & $<0.001$ & $<0.001$ & 0.001 & 0.001 & $<0.001$ & 0.001 & 0.014 & 0.294 & $<0.001$ & 0.004 & 0.040 & 0.814 \\
\hline Tin & 0.159 & 0.122 & 0.121 & 0.132 & 0.042 & 0.058 & 0.290 & 0.098 & 0.042 & 0.169 & $<0.050$ & 0.155 \\
\hline Titanium & 0.14 & 0.06 & 0.13 & 0.08 & 0.10 & 0.14 & 0.29 & 2.03 & 0.10 & 0.21 & 0.54 & 5.60 \\
\hline Tungsten & 0.001 & $<0.001$ & 0.002 & 0.003 & 0.001 & $<0.001$ & $<0.001$ & $<0.010$ & 0.001 & 0.003 & $<0.010$ & 0.039 \\
\hline Uranium & 0.0026 & 0.0025 & 0.0075 & 0.0238 & 0.0026 & 0.0062 & 0.0455 & 0.818 & 0.0026 & 0.0177 & 0.142 & 2.55 \\
\hline Vanadium & 0.02 & 0.02 & 0.03 & 0.04 & 0.03 & 0.03 & 0.10 & 1.39 & 0.03 & 0.06 & 0.37 & 7.30 \\
\hline Yttrium & 0.002 & 0.002 & 0.002 & 0.004 & 0.003 & 0.003 & 0.003 & 0.012 & 0.003 & 0.002 & $<0.010$ & 0.035 \\
\hline Zinc & 1.4 & 1.8 & 1.2 & 1.5 & 1.2 & 1.7 & 6.3 & 107 & 1.2 & 3.3 & 21.5 & 405 \\
\hline
\end{tabular}




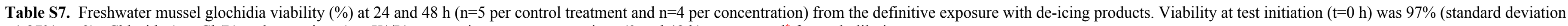
$=1.27 \%, \mathrm{n}=3)$. Chloride $\left(\mathrm{mg} \mathrm{Cl}^{-} / \mathrm{L}\right)$ and potassium $\left(\mathrm{mg} \mathrm{K}^{+} / \mathrm{L}\right)$ geometric mean concentrations $(0$ and $48 \mathrm{~h})$ are reported ${ }^{*}$ for each dilution treatment.

\begin{tabular}{|c|c|c|c|c|c|c|c|c|c|c|c|c|c|c|}
\hline \multirow[b]{2}{*}{ Dilution } & \multirow[b]{2}{*}{$\mathrm{Cl}-(\mathrm{mg} \mathrm{Cl} / \mathrm{L})$} & \multicolumn{2}{|l|}{ Brine } & \multirow[b]{2}{*}{ Viability $48 \mathrm{~h}$} & \multicolumn{5}{|c|}{ Brine-Beet Juice } & \multicolumn{5}{|c|}{ Beet Juice } \\
\hline & & $\mathbf{K}^{+}(\mathbf{m g ~ K} / \mathbf{L})^{*}$ & Viability $24 \mathrm{~h}$ & & Dilution (\%) & $\mathrm{Cl}-(\mathrm{mg} \mathrm{Cl} / \mathrm{L})$ & $\mathbf{K}+(\mathbf{m g ~ K} / \mathbf{L})$ & Viability $24 \mathrm{~h}$ & Viability $48 \mathrm{~h}$ & Dilution (\%) & $\mathrm{Cl}-(\mathrm{mg} \mathrm{Cl} / \mathrm{L})$ & $\mathbf{K}+(\mathbf{m g ~ K} / \mathbf{L})$ & Viability $24 \mathrm{~h}$ & Viability $48 \mathrm{H}$ \\
\hline 0.0 & 8.60 & 2.65 & 92.5 & 92.7 & 0.00 & 9.13 & 14.70 & 96.4 & 94.5 & 0.00 & 9.13 & 14.70 & 95.7 & 94.0 \\
\hline 0.0 & 8.60 & 2.65 & 92.9 & 92.5 & 0.00 & 9.13 & 1470 & 94.4 & 91.0 & 000 & 913 & 1470 & 985 & 948 \\
\hline 0.0 & 8.60 & 2.65 & 91.2 & 95.4 & 0.00 & 9.13 & 14.70 & 97.5 & 97.5 & 000 & 913 & 1470 & 94.4 & 973 \\
\hline 0.0 & $\begin{array}{l}8.00 \\
8.60\end{array}$ & 2.65 & 92.0 & $\begin{array}{l}93.4 \\
93.2\end{array}$ & 0.00 & 9.13 & 14.70 & 946 & 87.5 & 0.00 & 913 & 14.70 & $\begin{array}{l}34.4 \\
915\end{array}$ & 907 \\
\hline 0.0 & $\begin{array}{l}8.00 \\
8.60\end{array}$ & 2.65 & 93.9 & $\begin{array}{l}93.5 \\
97.5\end{array}$ & 0.00 & 9.13 & 14.70 & 94.8 & 95.2 & 0.00 & 9.13 & 14.70 & 97.3 & 96.3 \\
\hline 0.01 & $\begin{array}{l}0.00 \\
23.49\end{array}$ & 2.67 & 95.0 & 94.4 & 0.01 & 16.60 & 4.33 & 93.4 & 93.2 & 0.01 & 8.72 & 9.94 & 96.7 & 94.7 \\
\hline 0.01 & 23.49 & 2.67 & 99.3 & 94.7 & 0.01 & 16.60 & 4.33 & 91.0 & 91.3 & 0.01 & 8.72 & 9.94 & 84.6 & 83.6 \\
\hline 0.01 & 23.49 & 2.67 & 92.2 & 95.7 & 0.01 & 16.60 & 4.33 & 97.6 & 89.4 & 0.01 & 8.72 & 9.94 & 95.3 & 96.5 \\
\hline 0.01 & 23.49 & 2.67 & 98.5 & 97.4 & 0.01 & 16.60 & 4.33 & 97.8 & 97.4 & 0.01 & 8.72 & 9.94 & 95.0 & 95.2 \\
\hline 0.1 & 187.01 & 2.80 & 87.7 & 75.7 & 0.03 & 49.52 & 15.19 & 66.4 & 70.9 & 0.03 & $\begin{array}{l}0.12 \\
22.96\end{array}$ & 32.65 & 35.2 & 20.9 \\
\hline 0.1 & 187.01 & 2.80 & 90.1 & 81.7 & 0.03 & 49.52 & 15.19 & $\begin{array}{l}69.2 \\
69.2\end{array}$ & 61.7 & 0.03 & 22.96 & 32.65 & 21.0 & 20.5 \\
\hline 0.1 & 187.01 & 2.80 & 89.5 & 93.8 & 0.03 & 49.52 & 15.19 & 92.8 & 31.3 & 0.03 & 22.96 & 32.65 & 1.4 & 10.9 \\
\hline 0.1 & $\begin{array}{l}187.01 \\
\end{array}$ & 2.80 & 92.0 & 91.7 & 0.03 & 49.52 & 15.19 & 86.6 & 73.2 & 0.03 & 22.96 & 32.65 & 6.4 & 17.7 \\
\hline 0.3 & 523.14 & 2.96 & 64.5 & 54.7 & 0.1 & 89.89 & 21.83 & 61.2 & 63.0 & 0.1 & 28.16 & 46.06 & 2.6 & 17.2 \\
\hline 0.3 & 523.14 & 2.96 & 66.0 & 55.6 & 0.1 & 89.89 & 21.83 & 27.0 & 12.3 & 0.1 & 28.16 & 46.06 & 1.6 & 10.2 \\
\hline 0.3 & 523.14 & 2.96 & 60.3 & 67.5 & 0.1 & 89.89 & $\begin{array}{l}21.03 \\
21.83\end{array}$ & $\begin{array}{l}69.2 \\
69.2\end{array}$ & 16.2 & 0.1 & $\begin{array}{l}28.10 \\
28.16\end{array}$ & $\begin{array}{l}40.00 \\
46.06\end{array}$ & $\begin{array}{l}1.0 \\
0.7\end{array}$ & $\begin{array}{l}10.2 \\
5.8\end{array}$ \\
\hline 0.3 & 523.14 & 2.96 & 65.8 & 86.5 & 0.1 & 89.89 & 21.83 & 40.0 & $\begin{array}{l}10.2 \\
9.5\end{array}$ & $\begin{array}{l}0.1 \\
0.1\end{array}$ & $\begin{array}{l}20.10 \\
28.16\end{array}$ & $\begin{array}{l}0.0 .00 \\
46.06\end{array}$ & 21.3 & $\begin{array}{l}.0 \\
4.8\end{array}$ \\
\hline 0.5 & 853.42 & 3.00 & 60.2 & 38.8 & 0.1 & 152.97 & 23.36 & 10.0 & 0.0 & $\begin{array}{l}0.1 \\
0.1\end{array}$ & $\begin{array}{l}20.10 \\
45.96\end{array}$ & $\begin{array}{l}0.0 .00 \\
84.49\end{array}$ & 0.7 & $\begin{array}{l}.0 \\
0.0\end{array}$ \\
\hline 0.5 & 853.42 & 3.00 & 39.3 & 48.7 & 0.1 & 152.97 & 23.36 & $\begin{array}{l}10.0 \\
16.0\end{array}$ & 7.8 & $\begin{array}{l}0.1 \\
0.1\end{array}$ & $\begin{array}{l}5.90 \\
45.96\end{array}$ & $\begin{array}{l}\text { 40.49 } \\
84.49\end{array}$ & 0.0 & $\begin{array}{l}9.0 \\
9.2\end{array}$ \\
\hline 0.5 & 853.42 & 3.00 & 45.9 & 46.1 & 0.1 & 152.97 & 23.36 & 17.4 & 19.2 & 0.1 & 45.96 & 84.49 & 17.4 & 5.5 \\
\hline 0.5 & 853.42 & 3.00 & 62.0 & 47.3 & 0.1 & 152.97 & 23.36 & 0.9 & 19.6 & 0.1 & 45.96 & 84.49 & 0.0 & 5.4 \\
\hline 0.75 & 1284.52 & 3.32 & 39.5 & $\begin{array}{l}7+.5 \\
58.6\end{array}$ & $\begin{array}{l}0.1 \\
0.5\end{array}$ & 756.04 & $\begin{array}{l}2.50 \\
118.06\end{array}$ & 0.0 & 0.0 & 0.5 & 195.22 & 391.91 & 0.0 & 10.0 \\
\hline 0.75 & 1284.52 & 3.32 & 44.1 & 0.8 & 0.5 & $\begin{array}{l}750.04 \\
756.04\end{array}$ & 118.06 & 0.0 & 0.0 & 0.5 & 195.22 & 391.91 & 0.0 & 3.6 \\
\hline 0.75 & 1284.52 & 3.32 & $\begin{array}{l}44.1 \\
44.7\end{array}$ & $\begin{array}{l}.0 .0 \\
13.6\end{array}$ & 0.5 & $\begin{array}{l}756.04 \\
756.04\end{array}$ & $\begin{array}{l}110.00 \\
118.06\end{array}$ & 0.7 & 10.9 & 0.5 & 195.22 & 391.91 & 0.6 & 1.8 \\
\hline 0.75 & 1284.52 & 3.32 & 40.2 & 27.3 & 0.5 & 756.04 & 118.06 & 8.0 & 7.5 & 0.5 & 195.22 & 391.91 & 0.0 & $\begin{array}{l}1.0 \\
1.7\end{array}$ \\
\hline 1.0 & 1714.82 & 3.28 & $\begin{array}{l}10.2 \\
1.3\end{array}$ & 20.2 & 1.0 & $\begin{array}{l}1504.049 \\
1504\end{array}$ & $\begin{array}{l}110.00 \\
220.92\end{array}$ & $\begin{array}{l}0.0 \\
0.0\end{array}$ & 0.0 & 1.0 & 385.36 & 768.93 & 0.0 & 0.5 \\
\hline 1.0 & $\begin{array}{l}17144.82 \\
1714.82\end{array}$ & 3.28 & 1.8 & $\begin{array}{l}18.2 \\
18.3\end{array}$ & $\begin{array}{l}1.0 \\
1.0\end{array}$ & 1504.79 & 220.92 & 0.0 & 0.0 & 1.0 & 385.36 & 768.93 & 0.0 & 0.0 \\
\hline 1.0 & $\begin{array}{l}17144.82 \\
1714.82\end{array}$ & 3.28 & $\begin{array}{l}1.0 \\
3.9\end{array}$ & $\begin{array}{l}30.6 \\
38.6\end{array}$ & $\begin{array}{l}1.0 \\
1.0\end{array}$ & 1504.79 & 220.92 & 4.8 & 0.0 & 1.0 & 385.36 & 768.93 & 0.0 & 0.8 \\
\hline 1.0 & $\begin{array}{l}17144.82 \\
1714.82\end{array}$ & 3.28 & 1.2 & $\begin{array}{l}13.0 \\
13.2\end{array}$ & 1.0 & 1504.79 & 220.92 & 11.0 & 0.0 & 1.0 & 385.36 & 768.93 & 1.3 & 2.0 \\
\hline 2.0 & 3469.64 & 3.87 & 0.0 & 0.0 & 2.0 & 3099.85 & 408.82 & 0.0 & 0.0 & 2.0 & 774.30 & 1622.22 & 0.0 & 0.0 \\
\hline 2.0 & 3469.64 & 3.87 & 0.0 & 0.0 & 2.0 & 3099.85 & 408.82 & 0.0 & 0.0 & 2.0 & 774.30 & 1622.22 & 0.0 & 0.0 \\
\hline 2.0 & 3469.64 & 3.87 & 2.0 & 0.0 & 2.0 & 3099.85 & 408.82 & 8.9 & 0.6 & 2.0 & 774.30 & 1622.22 & 8.0 & 0.0 \\
\hline 2.0 & 3469.64 & 3.87 & 0.0 & 0.0 & 2.0 & 3099.85 & 408.82 & 7.8 & 0.9 & 2.0 & 774.30 & $\begin{array}{l}1022.22 \\
16222\end{array}$ & 0.0 & 3.9 \\
\hline
\end{tabular}

"Concentration of potassium $\left(\mathrm{mg} \mathrm{K}^{+} / \mathrm{L}\right)$ at $\mathrm{t}=0$ was reported for brine exposure 
Table S8. Summary of lethal (LC) and effect (EC) concentration data (with $95 \%$ confidence limits (CI) when available) for early life stage freshwater mussels (black text), early life stage marine mussels (blue text) and other freshwater adult bivalves (green text). Where $\mathrm{d}=\mathrm{day}$, $\mathrm{wk}=$ week, and $\mathrm{m}=$ month.

\begin{tabular}{|c|c|c|c|c|c|c|c|c|}
\hline Metals & Species & Life Stage & Exposure & Acute/Chronic & LC/EC50 & $95 \% \mathrm{CI}$ & Units & Reference \\
\hline Aluminum & Lampsilis siliquoidea & 7-8 d juvenile & $96-\mathrm{h}$ & acute & $>6200$ & - & $\mu \mathrm{g} \mathrm{Al} / \mathrm{L}$ & Wang et al., 2017 \\
\hline Aluminum & Lampsilis siliquoidea & 6-wk juvenile & $28-d$ & chronic & $>1103$ & - & $\mu \mathrm{g} \mathrm{Al} / \mathrm{L}$ & Wang et al., 2017 \\
\hline Aluminum & Margaritifera margaritifera & 13-28-m juvenile & 96-h & acute & $>954$ & - & $\mu \mathrm{g} \mathrm{Al} / \mathrm{L}$ & Belamy et al., 2020 \\
\hline Arsenic & Margaritifera margaritifera & 13-28-m juvenile & 96-h & acute & $>127$ & - & $\mu \mathrm{g} \mathrm{As} / \mathrm{L}$ & Belamy et al., 2020 \\
\hline Boron & Lampsilis siliquoidea & $<5$-d juvenile & 96-h & acute & 137 & $86-200$ & $\mathrm{mg} \mathrm{B} / \mathrm{L}$ & Soucek et al., 2011 \\
\hline Boron & Ligumia recta & $<5$-d juvenile & 96-h & acute & 147 & $87-246$ & $\mathrm{mg} \mathrm{B} / \mathrm{L}$ & Soucek et al., 2011 \\
\hline Boron & Megalonaias nervosa & $<5$-d juvenile & 96-h & acute & $>544$ & - & $\mathrm{mg} \mathrm{B} / \mathrm{L}$ & Soucek et al., 2011 \\
\hline Boron & Lampsilis siliquoidea & 3-m juvenile & $21-d$ & chronic & 38.4 & $37.1-39.5$ & $\mathrm{mg} \mathrm{B} / \mathrm{L}$ & Hall et al., 2014 \\
\hline Cadmium & Margaritifera margaritifera & 13-28-m juvenile & 96-h & acute & $>110$ & - & $\mu \mathrm{g} \mathrm{Cd} / \mathrm{L}$ & Belamy et al., 2020 \\
\hline Cadmium & Lampsilis siliquoidea & glochidia & 48-h & acute & $>227$ & - & $\mu \mathrm{g} \mathrm{Cd} / \mathrm{L}$ & Wang et al., 2010 \\
\hline Cadmium & Lampsilis siliquoidea & 5-d juvenile & 96-h & acute & 16 & $13-20$ & $\mu \mathrm{g} \mathrm{Cd} / \mathrm{L}$ & Wang et al., 2010 \\
\hline Cadmium & Lampsilis siliquoidea & 2-m juvenile & 96-h & acute & $>62$ & - & $\mu \mathrm{g} \mathrm{Cd} / \mathrm{L}$ & Wang et al., 2010 \\
\hline Cadmium & Lampsilis siliquoidea & 6-m juvenile & 96-h & acute & 199 & $118-337$ & $\mu \mathrm{g} \mathrm{Cd} / \mathrm{L}$ & Wang et al., 2010 \\
\hline Cadmium & Lampsilis siliquoidea & 2-4-m juvenile & $28-d$ & chronic & 8.1 & $7.2-9.2$ & $\mu \mathrm{g} \mathrm{Cd} / \mathrm{L}$ & Wang et al., 2010 \\
\hline Cadmium & Anodonta imbecilis & 24-48-m juvenile & 48-h & acute & 0.137 & - & $\mu \mathrm{g} \mathrm{Cd} / \mathrm{L}$ & Keller and Zam, 1991 \\
\hline Cadmium & Anodonta imbecilis & 24-48-m juvenile & 96-h & acute & 0.107 & - & $\mu \mathrm{g} \mathrm{Cd} / \mathrm{L}$ & Keller and Zam, 1991 \\
\hline Cadmium & Anodonta anatina & glochidia & 24-h & acute & 755 & - & $\mu \mathrm{g} \mathrm{Cd} / \mathrm{L}$ & Hansten et al., 1996 \\
\hline Chromium & Anodonta imbecilis & 24-48-m juvenile & 48-h & acute & 1.187 & - & $\mathrm{mg} \mathrm{Cr} / \mathrm{L}$ & Keller and Zam, 1991 \\
\hline Chromium & Anodonta imbecilis & 24-48-m juvenile & 96-h & acute & 0.618 & - & $\mathrm{mg} \mathrm{Cr} / \mathrm{L}$ & Keller and Zam, 1991 \\
\hline Copper & Lampsilis siliquoidea & glochidia & 48-h & acute & 26 & $25-27$ & $\mu \mathrm{g} \mathrm{Cu} / \mathrm{L}$ & Wang et al., 2010 \\
\hline Copper & Lampsilis siliquoidea & 5-d juvenile & 96-h & acute & 20 & $17-23$ & $\mu \mathrm{g} \mathrm{Cu} / \mathrm{L}$ & Wang et al., 2010 \\
\hline Copper & Lampsilis siliquoidea & 2-m juvenile & 96-h & acute & 35 & $27-47$ & $\mu \mathrm{g} \mathrm{Cu} / \mathrm{L}$ & Wang et al., 2010 \\
\hline Copper & Anodonta anatina & glochidia & $24-\mathrm{h}$ & acute & 43 & - & $\mu \mathrm{g} \mathrm{Cu} / \mathrm{L}$ & Hansten et al., 1996 \\
\hline Copper & Epioblasma torulosa & glochidia & $48-\mathrm{h}$ & acute & 8.7 & $6.7-10.7$ & $\mu \mathrm{g} \mathrm{Cu} / \mathrm{L}$ & Gillis et al., 2008 \\
\hline Copper & Epioblasma triquetra & glochidia & 48-h & acute & 5.2 & $4.7-5.7$ & $\mu \mathrm{g} \mathrm{Cu} / \mathrm{L}$ & Gillis et al., 2008 \\
\hline Copper & Lampsilis fasciola & glochidia & $48-\mathrm{h}$ & acute & 12.5 & $11.6-13.6$ & $\mu \mathrm{g} \mathrm{Cu} / \mathrm{L}$ & Gillis et al., 2008 \\
\hline Copper & Lampsilis siliquoidea & glochidia & 48-h & acute & 21.6 & $18.8-25.5$ & $\mu \mathrm{g} \mathrm{Cu} / \mathrm{L}$ & Gillis et al., 2008 \\
\hline Copper & Ortmanniana subrotunda & glochidia & 48-h & acute & 11.3 & $9.3-13.9$ & $\mu \mathrm{g} \mathrm{Cu} / \mathrm{L}$ & Gillis et al., 2008 \\
\hline Copper & Villosa fabalis & glochidia & 48-h & acute & 4.6 & $3.7-5.6$ & $\mu \mathrm{g} \mathrm{Cu} / \mathrm{L}$ & Gillis et al., 2008 \\
\hline Copper & Villosa iris & glochidia & $24-\mathrm{h}$ & acute & 47 & - & $\mu \mathrm{g} \mathrm{Cu} / \mathrm{L}$ & Jacobson et al., 1997 \\
\hline Copper & Pyganadon grandis & glochidia & $24-h$ & acute & 46 & - & $\mu \mathrm{g} \mathrm{Cu} / \mathrm{L}$ & Jacobson et al., 1997 \\
\hline Copper & Anodonta imbecilis & 24-48-m juvenile & 48-h & acute & 0.388 & - & $\mu \mathrm{g} \mathrm{Cu} / \mathrm{L}$ & Keller and Zam, 1991 \\
\hline Copper & Anodonta imbecilis & 24-48-m juvenile & 96-h & acute & 0.199 & - & $\mu \mathrm{g} \mathrm{Cu} / \mathrm{L}$ & Keller and Zam, 1991 \\
\hline Copper & Lampsilis cardium & glochidia & 24-h & acute & 0.21 & - & $\mathrm{mg} \mathrm{Cu} / \mathrm{L}$ & Milam et al., 2003 \\
\hline Copper & Lampsilis siliquoidea & glochidia & 24-h & acute & 0.13 & - & $\mathrm{mg} \mathrm{Cu} / \mathrm{L}$ & Milam et al., 2003 \\
\hline Copper & Ligumia subrostrata & glochidia & 24-h & acute & 0.15 & - & $\mathrm{mg} \mathrm{Cu} / \mathrm{L}$ & Milam et al., 2003 \\
\hline Copper & Utterbackia imbecillis & glochidia & $24-\mathrm{h}$ & acute & 0.52 & - & $\mathrm{mg} \mathrm{Cu} / \mathrm{L}$ & Milam et al., 2003 \\
\hline Copper & Lampsilis siliquoidea & juveniles & $28-d$ & chronic & 7.27 & $5.96-8.86$ & $\mu \mathrm{g} \mathrm{Cu} / \mathrm{L}$ & Jorge et al., 2013 \\
\hline Lead & Lampsilis siliquoidea & glochidia & 48-h & acute & $>400$ & - & $\mu \mathrm{g} \mathrm{Pb} / \mathrm{L}$ & Wang et al., 2010 \\
\hline Lead & Lampsilis siliquoidea & 5-d juvenile & 96-h & acute & $142(124-164)$ and & $8(249-357)$ & $\mu \mathrm{g} \mathrm{Pb} / \mathrm{L}$ & Wang et al., 2010 \\
\hline Lead & Lampsilis siliquoidea & 5-d juvenile & 96-h & acute & 142(124-164) and & $8(249-357)$ & $\mu \mathrm{g} \mathrm{Pb} / \mathrm{L}$ & Wang et al., 2010 \\
\hline Lead & Lampsilis siliquoidea & 2-m juvenile & 96-h & acute & $>426$ & - & $\mu \mathrm{g} \mathrm{Pb} / \mathrm{L}$ & Wang et al., 2010 \\
\hline Lead & Lampsilis siliquoidea & 2-4-m juvenile & $28-d$ & chronic & 20 & $17-43$ & $\mu \mathrm{g} \mathrm{Pb} / \mathrm{L}$ & Wang et al., 2010 \\
\hline Lithium & Mytilus galloprovincialis & $2.25-3.5 \mathrm{~cm}$ & $9-d$ & chronic & 153.78 & - & $\mathrm{mg} \mathrm{Li} / \mathrm{L}$ & Fraga, 2020 \\
\hline Manganese & Mytilus edulis & larvae & $48-h$ & acute & 30 & $40-46$ & $\mathrm{mg} \mathrm{Mn} / \mathrm{L}$ & Morgan et al., 1986 \\
\hline Molybdenum & Mytilus edulis & larvae & 48-h & acute & 147 & $127-169$ & $\mathrm{mg} \mathrm{Mo} / \mathrm{L}$ & Morgan et al., 1986 \\
\hline Nickel & Anodonta imbecilis & 24-48-m juvenile & 48-h & acute & 0.471 & - & $\mathrm{mg} \mathrm{Ni} / \mathrm{L}$ & Keller and Zam, 1991 \\
\hline Nickel & Anodonta imbecilis & 24-48-m juvenile & 96-h & acute & 0.252 & - & $\mathrm{mg} \mathrm{Ni} / \mathrm{L}$ & Keller and Zam, 1991 \\
\hline Uranium & Corbicula sp. & $2-2.5 \mathrm{~cm}$ adults & 96-h & acute & 1872.08 & $228-3516$ & $\mathrm{mg} \mathrm{U} / \mathrm{L}$ & Labrot et al., 1999 \\
\hline Zinc & Villosa iris & glochidia & 48-h & acute & $577-4123$ & & $\mu \mathrm{g} \mathrm{Zn/L}$ & McCann, 1993 \\
\hline Zinc & Villosa iris & juveniles & 48-h & acute & $360-1885$ & & $\mu \mathrm{g} \mathrm{Zn} / \mathrm{L}$ & McCann, 1993 \\
\hline Zinc & Lampsilis fasciola & glochidia & 48-h & acute & 688 & & $\mu \mathrm{g} \mathrm{Zn} / \mathrm{L}$ & Gillis et al., 2021 \\
\hline Zinc & Lampsilis siliquoidea & glochidia & 48-h & acute & $>200$ and $>964$ & - & $\mu \mathrm{g} \mathrm{Zn} / \mathrm{L}$ & Wang et al., 2010 \\
\hline Zinc & Lampsilis siliquoidea & 5-d juvenile & 96-h & acute & $151(131-173)$ anc & $75(155-198)$ & $\mu \mathrm{g} \mathrm{Zn} / \mathrm{L}$ & Wang et al., 2010 \\
\hline Zinc & Lampsilis siliquoidea & 2-m juvenile & 96-h & 48-h & $>471$ & - & $\mu \mathrm{g} \mathrm{Zn} / \mathrm{L}$ & Wang et al., 2010 \\
\hline Zinc & Lampsilis siliquoidea & 6-m juvenile & 96-h & 48-h & 1700 & $1200-2400$ & $\mu \mathrm{g} \mathrm{Zn} / \mathrm{L}$ & Wang et al., 2010 \\
\hline Zinc & Lampsilis siliquoidea & 2-4-m juvenile & $28-d$ & chronic & 333 & $271-410$ & $\mu \mathrm{g} \mathrm{Zn/L}$ & Wang et al., 2010 \\
\hline Zinc & Lampsilis siliquoidea & 2-4-m juvenile & $28-d$ & chronic & 228 & $202-256$ & $\mu \mathrm{g} \mathrm{Zn} / \mathrm{L}$ & Wang et al., 2010 \\
\hline Zinc & Anodonta anatina & glochidia & 24-h & acute & 346 & - & $\mu \mathrm{g} \mathrm{Zn} / \mathrm{L}$ & Hansten et al., 1996 \\
\hline Zinc & Anodonta imbecilis & 24-48-m juvenile & 48-h & acute & 0.588 & - & $\mu \mathrm{g} \mathrm{Zn} / \mathrm{L}$ & Keller and Zam, 1991 \\
\hline Zinc & Anodonta imbecilis & 24-48-m juvenile & 96-h & acute & 0.438 & - & $\mu \mathrm{g} \mathrm{Zn} / \mathrm{L}$ & Keller and Zam, 1991 \\
\hline
\end{tabular}




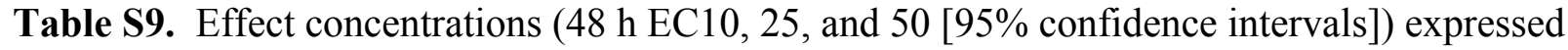
as the concentration of chloride (geometric mean of $\mathrm{t}=24$ and $48 \mathrm{~h}$ ) or potassium for the sodium chloride reference test and de-icing products in an exposure with freshwater mussel larvae (glochidia).

\begin{tabular}{lcccccc}
\hline & \multicolumn{2}{c}{ EC10 } & \multicolumn{2}{c}{ EC25 } & \multicolumn{2}{c}{ EC50 } \\
\multicolumn{1}{c}{ Product } & $\begin{array}{c}\text { Chloride } \\
(\mathbf{m g} / \mathbf{L})\end{array}$ & $\begin{array}{c}\text { Potassium } \\
(\mathbf{m g} / \mathbf{L})\end{array}$ & $\begin{array}{c}\text { Chloride } \\
(\mathbf{m g} / \mathbf{L})\end{array}$ & $\begin{array}{c}\text { Potassium } \\
(\mathbf{m g} / \mathbf{L})\end{array}$ & $\begin{array}{c}\text { Chloride } \\
(\mathbf{m g} / \mathbf{L})\end{array}$ & $\begin{array}{c}\text { Potassium } \\
(\mathbf{m g} / \mathbf{L})\end{array}$ \\
\hline \multirow{2}{*}{ NaCl Reference } & 400 & - & 629 & - & 991 & - \\
& $(270-503)$ & & $(528-732)$ & & $(880-1102)$ & \\
Brine & 199 & - & 382 & - & 732 & - \\
& $(267-497)$ & & $(267-497)$ & & $(610-854)$ & \\
Brine-Beet Juice & 19.7 & 13.0 & 33.4 & 15.3 & 56.7 & 18.0 \\
& $(10.4-28.9)$ & $(11.6-14.4)$ & $(23.6-43.1)$ & $(16.8-19.1)$ & $(47.3-66.0)$ & $(16.8-19.1)$ \\
Beet Juice & 9.9 & 15.5 & 12.7 & 19.5 & 16.4 & 24.4 \\
& $(9.0-10.9)$ & $(13.6-17.5)$ & $(11.9-13.7)$ & $(17.7-21.2)$ & $(15.5-17.3)$ & $(22.9-26.0)$ \\
\hline
\end{tabular}


Table S10. Ontario surface water concentrations of potassium for 2010 to 2019 (reported as mg $\mathrm{K}^{+} / \mathrm{L}$ ). Water samples were collected from long-term monitoring sites throughout Ontario through the Provincial Water Quality Monitoring Network.

\begin{tabular}{|c|c|c|c|c|c|c|c|}
\hline Year & $\begin{array}{c}\text { Average } \\
{[\mathbf{K}+]}\end{array}$ & $\begin{array}{l}\text { Standard } \\
\text { Deviation }\end{array}$ & $\underset{[\mathbf{K}+]}{\operatorname{Minimum}}$ & $\underset{[\mathrm{K}+]}{\operatorname{Maximum}}$ & $\begin{array}{c}\text { Total } \\
\text { Sampling } \\
\text { Events }\end{array}$ & $\begin{array}{c}\text { Sampling Events } \\
{[\mathrm{K}+]>\text { EC10* }}\end{array}$ & $\begin{array}{c}\text { \% Sampling Events } \\
{[\mathrm{K}+]>\text { EC10* }}\end{array}$ \\
\hline 2010 & 2.8 & 4.4 & 0.1 & 100 & 2026 & 61 & 3.0 \\
\hline 2011 & 3.0 & 4.7 & 0.1 & 102 & 2185 & 74 & 3.4 \\
\hline 2012 & 3.2 & 5.5 & 0.2 & 105 & 2123 & 90 & 4.2 \\
\hline 2113 & 2.7 & 3.7 & 0.2 & 69.9 & 1838 & 42 & 2.3 \\
\hline 2014 & 2.6 & 2.9 & 0.2 & 60.3 & 2058 & 51 & 2.5 \\
\hline 2015 & 2.7 & 3.9 & 0.3 & 70.5 & 1690 & 55 & 3.3 \\
\hline 2016 & 2.6 & 3.7 & 0.1 & 68.2 & 1652 & 61 & 3.7 \\
\hline 2017 & 2.6 & 3.0 & 0.0 & 41.1 & 2181 & 75 & 3.4 \\
\hline 2018 & 2.7 & 3.4 & 0.2 & 61.4 & 2244 & 76 & 3.4 \\
\hline 2019 & 2.9 & 3.1 & 0.0 & 40.2 & 1673 & 71 & 4.2 \\
\hline
\end{tabular}

*EC10 reported in Salerno et al., 2019 for Villosa iris glochidia (7.77 mg K/L [4.40-11.13]). 
Table S11. Summary of potassium water quality guidelines and screening values for the protection of freshwater aquatic life.

A. Available water quality guidelines for potassium according to the 'Database on Environmental Quality Guidelines' (considered up to date as of December 31, 2012).

https://open.canada.ca/data/en/dataset/ece7204f-c486-4ed9-b318-ddf61935f02c

\begin{tabular}{|c|c|c|c|c|c|c|c|c|}
\hline $\begin{array}{l}\text { Chemical } \\
\text { Name }\end{array}$ & Media & $\begin{array}{l}\text { Guideline } \\
\text { Value } \\
(\mathrm{mg} / \mathrm{L})\end{array}$ & $\begin{array}{l}\text { Acute / } \\
\text { Chronic }\end{array}$ & $\begin{array}{l}\text { Protected } \\
\text { Receptors }\end{array}$ & $\begin{array}{l}\text { Narrative } \\
\text { Protection } \\
\text { Goal }\end{array}$ & $\begin{array}{l}\text { Operational } \\
\text { Protection } \\
\text { Goal }\end{array}$ & $\begin{array}{l}\text { Main } \\
\text { Jurisdiction }\end{array}$ & Reference \\
\hline Potassium & Water & 53 & Chronic & $\begin{array}{l}\text { All } \\
\text { aquatic } \\
\text { organisms }\end{array}$ & $\begin{array}{l}\text { No } \\
\text { unacceptable } \\
\text { effects to } \\
\text { aquatic } \\
\text { organisms } \\
\text { with less than } \\
1 \text { hour } \\
\text { exposure }\end{array}$ & $\begin{array}{l}\text { One half of the } \\
\text { geometric } \\
\text { mean of genus } \\
\text { EC50 values } \\
\text { divided by UF } \\
\text { (data } \\
\text { dependent) }\end{array}$ & $\begin{array}{l}\text { USA/Canada } \\
\text { (Great Lakes } \\
\text { Initiative); } \\
\text { EPA Region } 3\end{array}$ & $\begin{array}{l}\text { EPA, } \\
1995^{\mathrm{a}} \\
\mathrm{EPA} \\
2005^{\mathrm{b}}\end{array}$ \\
\hline
\end{tabular}

B. Probable no-effect concentration (PNEC) for potassium derived (final column) from the European Chemicals Agency Dossier for potassium chloride ${ }^{c}$.

\begin{tabular}{llllll}
\hline $\begin{array}{l}\text { Chemical } \\
\text { Name }\end{array}$ & $\begin{array}{l}\text { Hazard for } \\
\text { aquatic } \\
\text { organisms }\end{array}$ & $\begin{array}{l}\text { Hazard } \\
\text { assessment } \\
\text { conclusion }\end{array}$ & $\begin{array}{l}\text { KCI PNEC } \\
\text { freshwater }\end{array}$ & $\begin{array}{l}\text { KCI PNEC } \\
\text { freshwater for } \\
\text { intermittent } \\
\text { releases }\end{array}$ & $\begin{array}{l}\mathrm{K}^{+} \text {PNEC } \\
\text { freshwater }\end{array}$ \\
\hline $\begin{array}{l}\text { Potassium } \\
\text { Chloride }\end{array}$ & Freshwater & $\begin{array}{l}\text { PNEC aqua } \\
\text { (freshwater) }\end{array}$ & $0.1 \mathrm{mg} / \mathrm{L}$ & $1 \mathrm{mg} / \mathrm{L}$ & $0.052 \mathrm{mg} / \mathrm{L}$ \\
\hline
\end{tabular}

aEPA (Environmental Protection Agency), 1995. Great Lakes Region Initiative (Canada/USA) Surface Water, www.epa.gov/owow/tmdl/glsprohibit.pdf (outdated, i.e., unable to locate)

bEPA 2005, US Environmental Protection Agency, Region 3, Surface Water, https://www.epa.gov/risk/freshwater-screening-benchmarks. Original source: Suter, G.W. II, and Tsao, C.L. 1996. Toxicological Benchmarks for Screening Potential Contaminants of Concern for Effects on Aquatic Biota: 1996 Revision. ES/ER/TM-96/R2. Available at: http://www.esd.ornl.gov/programs/ecorisk/documents/tm96r2.pdf

${ }^{c}$ European Chemicals Agency https://echa.europa.eu/registration-dossier/-/registereddossier/14341 


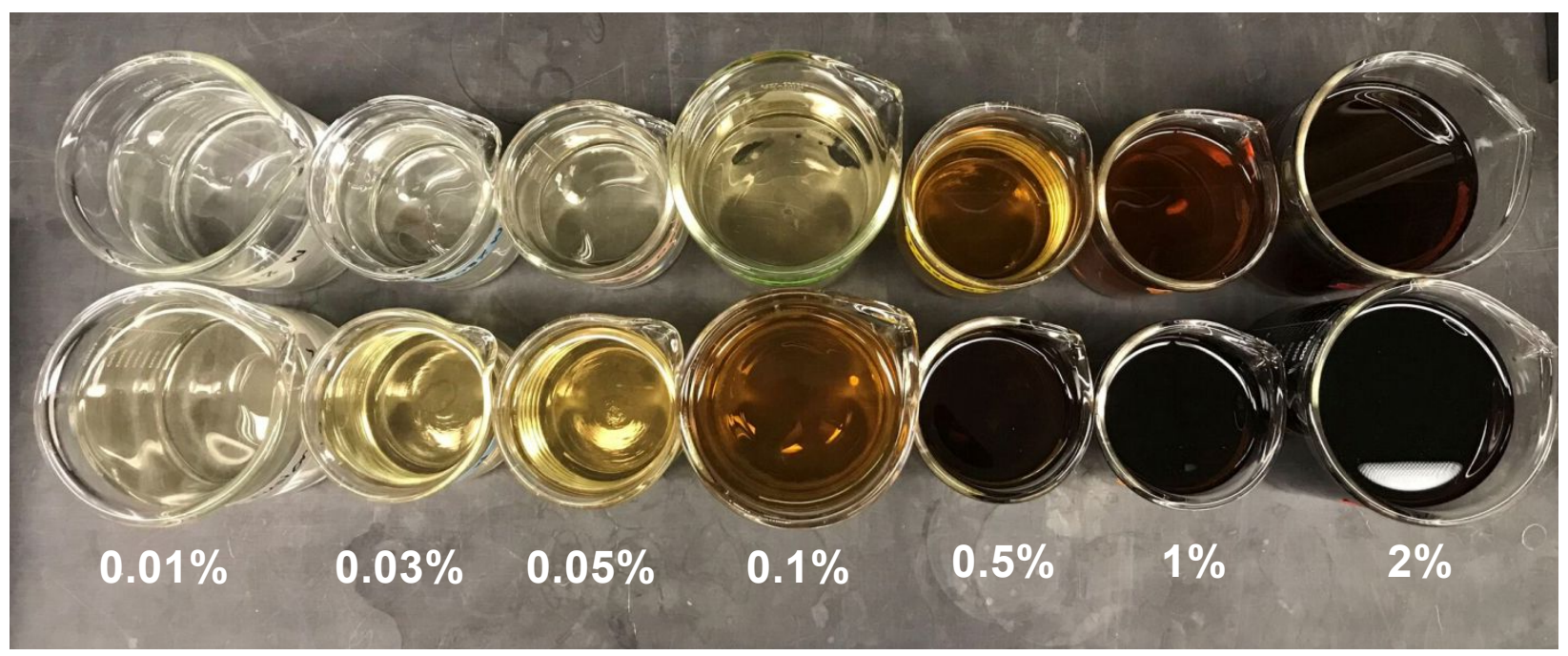

Figure S1. Exposure solutions used in acute toxicity tests with freshwater mussel larvae and road salt alternatives. The top row of beakers are dilutions of the brine-beet juice product and the bottom are dilutions of the beet juice de-icing product. 
A

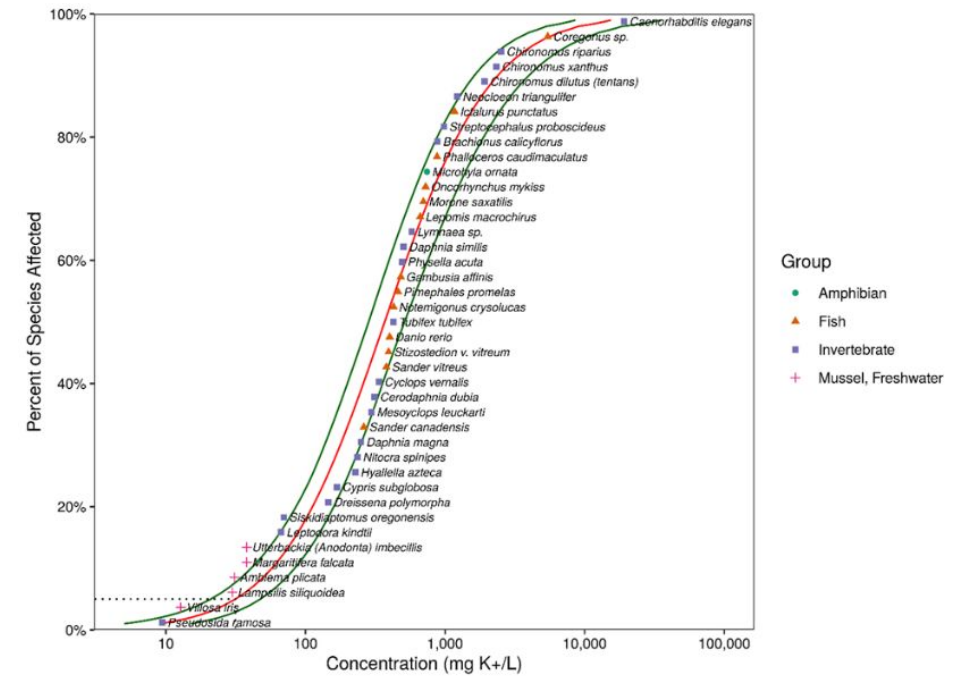

B

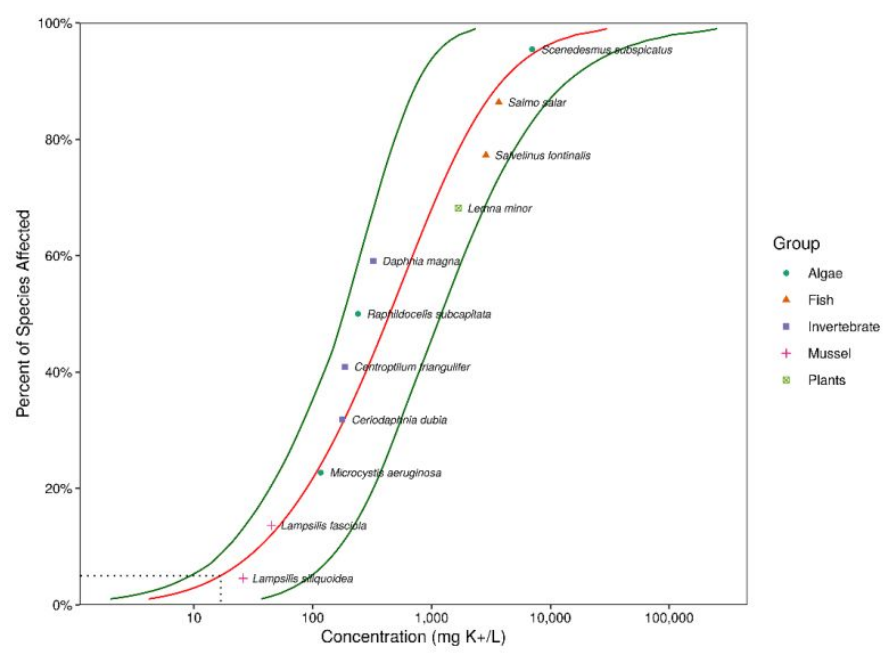

Figure S2. Species sensitivity distributions (SSD) of potassium toxicity for freshwater organisms based on published reports of LC/EC50 data for the acute-SSD (A), and no-effect and low-effect data for the chronic-SSD (B). The acute SSD revealed that a concentration of $31.5 \mathrm{mg} \mathrm{K}^{+} / \mathrm{L}$ (lower confidence limit (LCL) 15.7, upper confidence limit (UCL) 66.7) would result in acute toxicity (i.e. lethality) for $5 \%$ of previously studied organisms, and chronic effects, including reduced growth and reproduction have been reported in $5 \%$ of studied species at $6.8 \mathrm{mg} \mathrm{K}^{+} / \mathrm{L}$ (LCL 3.53 , UCL 121). 


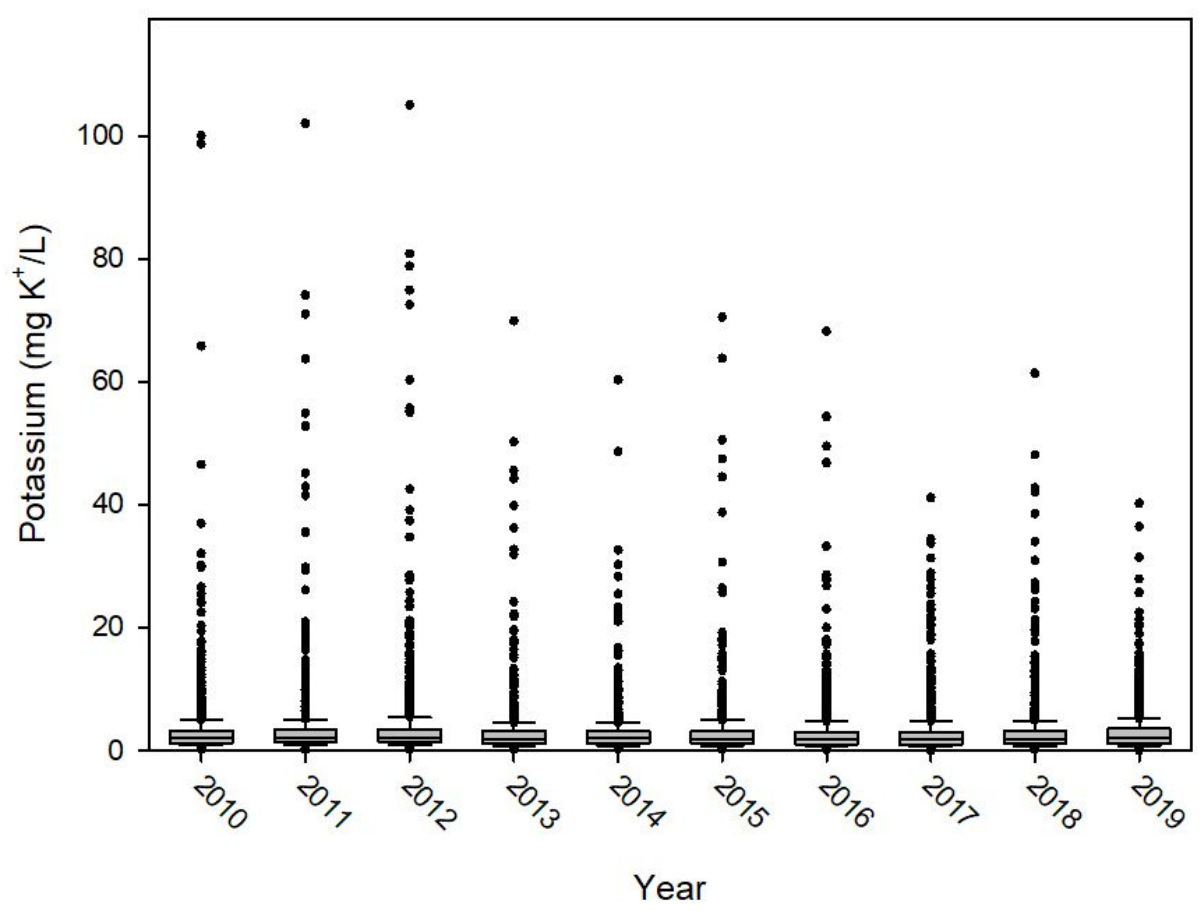

Figure S3. Box and whisker plot of Ontario surface water concentrations of potassium (reported as $\mathrm{mg} \mathrm{K}^{+} / \mathrm{L}$ ) from 2010 to 2019 . The median, first quartile ( $25^{\text {th }}$ percentile), third quartile $\left(75^{\text {th }}\right.$ percentile), $10^{\text {th }}$ and $90^{\text {th }}$ percentile along with outliers for each year are shown. Water samples were collected from long-term monitoring sites throughout Ontario through the Provincial Water Quality Monitoring Network (PWQMN). 


\section{Appendix A}

Input and output data for calculating effect concentrations (EC10, 25, and 50) using the drc package in RStudio ( $48 \mathrm{~h}$ glochidia viability results from $\mathrm{NaCl}$ reference test)

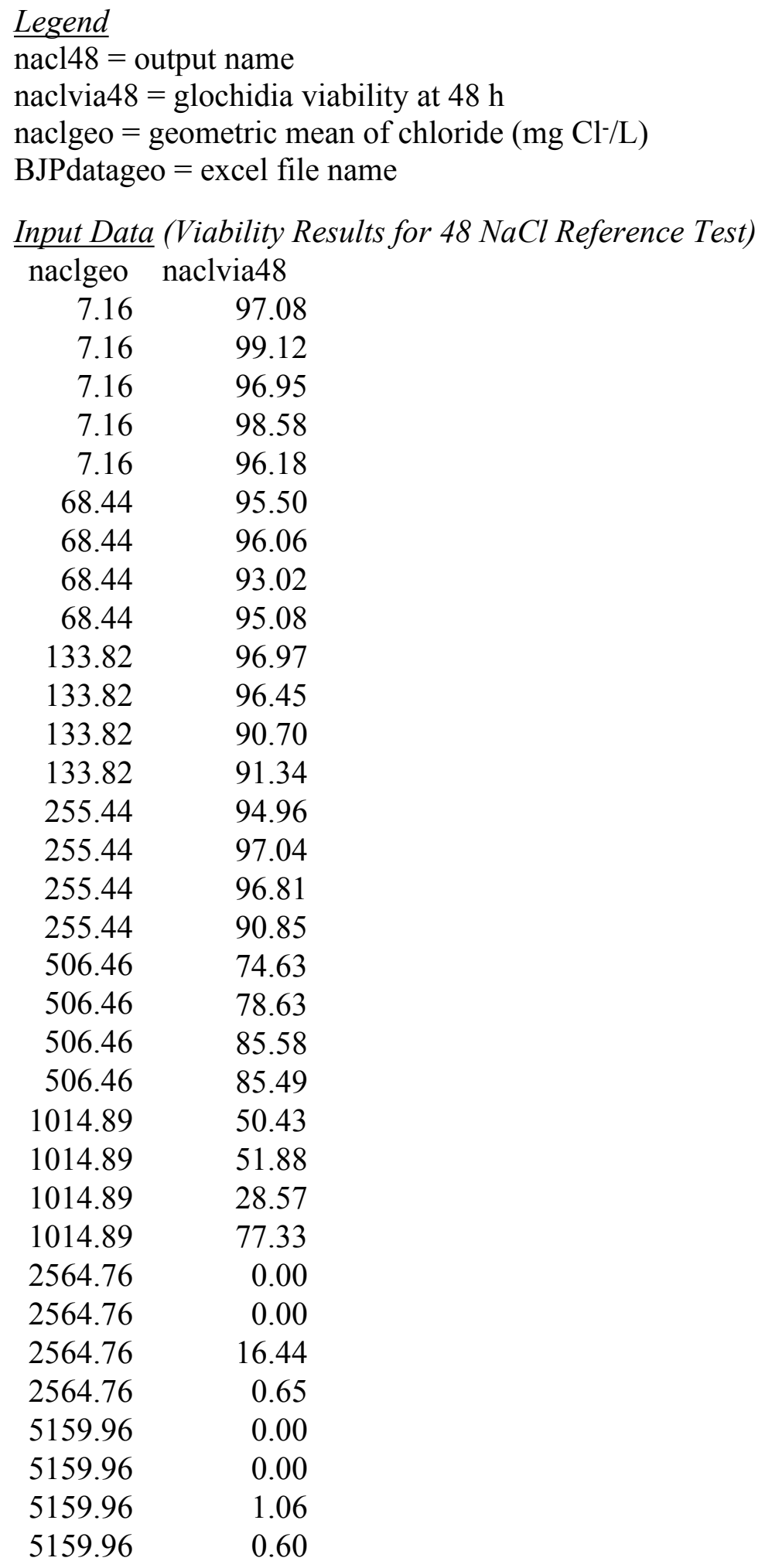


$>$ nacl48<-drm(naclvia48 naclgeo, data $=$ BJPdatageo, $\mathrm{fct}=$ LL.4(fixed $=\mathrm{c}(\mathrm{NA}, 0,100, \mathrm{NA})))$

$>$ summary(nacl48)

Output Data (Results)

Model fitted: Log-logistic (ED50 as parameter) (2 parms)

Parameter estimates:

$\begin{array}{lcllc} & \text { Estimate Std. } & \text { Error } & \text { t-value } & \text { p-value } \\ \text { b:(Intercept) } & 2.42026 & 0.30042 & 8.0562 & 4.258 \mathrm{e}-09 * * * \\ \text { e:(Intercept) } & 991.43622 & 54.53097 & 18.1812 & <2.2 \mathrm{e}-16 * * *\end{array}$

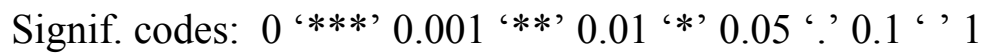

Residual standard error:

7.974715 (31 degrees of freedom)

$>\operatorname{ED}($ nacl48,c(10,25,50), interval $=$ "delta")

Estimated effective doses

Estimate Std. Error Lower Upper

e: $1: 10399.937 \quad 50.520 \quad 296.901502 .973$

e: $1: 25 \quad 629.692 \quad 50.044 \quad 527.626731 .758$

e: $1: 50991.436 \quad 54.531 \quad 880.2201102 .653$ 